\title{
UN ESTUDIO NORMATIVO DE LOS ÍTEMES MADURATIVOS DEL TEST DEL DIBUJO DE DOS FIGURAS HUMANAS EN NIÑOS INDÍGENAS YAQUIS*
}

\section{DEVELOPMENTAL ITEMS OF THE TWO HUMAN FIGURES TEST IN A SAMPLE OF YAQUI INDIAN CHILDREN. A NORMATIVE STUDY}

\author{
María Teresa Fernández Nistal**, Ana María Tuset Bertran ${ }^{* * *} Y$ \\ ENEIDA OCHOA AVILA****
}

\author{
*Estudio financiado por el Programa de Fomento y Apoyo a Proyectos de Investigación del Instituto \\ Tecnológico de Sonora - México (Clave: 00288). \\ **Doctora en Psicología. Profesora Investigadora Titular C del Departamento de Psicología del Instituto \\ Tecnológico de Sonora, México. E-Mail: teresa.fernandez@itson.edu.mx \\ ***Doctora en Psicología. Profesora Emérita del Departamento de Personalidad, Evaluación y Tratamiento \\ Psicológicos. Universidad de Barcelona, España.

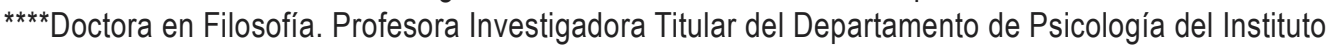 \\ Tecnológico de Sonora, México. \\ Instituto Tecnológico de Sonora. Calle 5 de Febrero, 818 Sur. Col. Centro, 85000, Ciudad Obregón, Sonora, México.
}

\section{RESUMEN}

Los objetivos del estudio que se informa fueron analizar las propiedades psicométricas del Test del Dibujo de Dos Figuras Humanas - Madurativo (T2F-M) de Maganto y Garaigordobil (2009a) y obtener normas de este test en una muestra de 654 niños del grupo indígena yaqui (México) de 5 a 11 años de edad. El análisis de la validez se realizó a través del estudio evolutivo de los ítemes madurativos y de la correlación entre el funcionamiento de los niños en el T2F-M y el Test de Matrices Progresivas Coloreadas (MPC) de Raven (1993). En cuanto a la fiabilidad, se estudió la consistencia interna de los ítemes evolutivos y el acuerdo interevaluadores. Las normas específicas se compararon con las de Maganto y Garaigordobil (2009a) para determinar la adecuación de estas normas en la población yaqui. Los resultados indicaron: (a) diferencias estadísticamente signi- ficativas en el número total de ítemes madurativos según la edad de los niños, con un aumento en la media de ítemes presentes en los dibujos a medida que se incrementa su edad, (b) una correlación positiva significativa de baja a moderada entre el funcionamiento en el T2F-M y el MPC, (c) una consistencia interna muy alta entre los ítemes del test, (d) un acuerdo intercalificadores muy alto y (e) diferencias entre las normas de puntuación obtenidas en la muestra de niños yaquis y las de Maganto y Garaigordobil (2009a). Se concluye que el T2F$\mathrm{M}$ es útil para evaluar el nivel de madurez mental de los niños de esta comunidad indígena, pero con el uso de normas locales que aseguren la realización de interpretaciones correctas de las puntuaciones que obtienen estos niños.

Palabras clave: Dibujo de la Figura Humana; Indígenas yaquis; Madurez mental; Validez; Normas. 


\section{ABSTRACT}

The study aims to analyze the psychometric properties of the developmental items of the Two Human Figures Test (T2F-M - Maganto \& Garaigordobil, 2009a) and to obtain standards of this test on a sample of 654 children from the Yaqui indigenous group (Mexico) between 5 and 11 years old. The validity analysis was performed using the developmental study of items and the correlation between the performance of children at T2F-M and the Coloured Progressive Matrices Test (CPM) (Raven, 1993). The internal consistency of developmental items and interrater agreement was studied to determine reliability. In order to obtain the T2F-M norms of the Yaqui children sample, the criterion of assignment of standardized scores of Maganto and Garaigordobil (2009a) was applied to the data obtained in this study, to each of the developmental items of both human figures (feminine and masculine) and for the different age groups. That is, items with a frequency of $85 \%$ or more were classified in category 1 , items with a frequency between 84 and $50 \%$ in category 2 , items with a frequency between 49 and $15 \%$ in category 3 and items with a frequency of $14 \%$ or less in category 4. Specific rules were compared with those of Maganto and Garaigordobil (2009a) in order to determine the suitability of these standards to the Yaqui population. The results on the psychometric qualities of the T2F-M indicate: (a) statistically significant differences in the total number of developmental items according to children's age $[F(6,654)=100.374 ; p<.01]$, the mean of items present in the two drawings increases with children's age, from 27.52 for the 5 years old to 59.20 for the 11 years old, (b) significant positive correlation, low to moderate, between performance in the T2F-M and the CPM; in most age groups the correlation between T2F-M and the CPM was significant, positive and with low values (between .211 and .382), except for the age group of 7 years, where the correlation was not significant; considering the total sample, the correlation was positive and significant and presented a moderate value (.608); (c) high internal consistency among test items; Cronbach Alpha coefficients and Spearman-Brown in the different age groups ranging from high (.814) and very high (.922); in the total sample, both coefficients were very high $(.950$ and .939 , respectively), (d) high interrater agreement (.993). Regarding the second objective of this work, obtaining specific rules in the T2F-M and comparing them with those of Maganto and Garaigordobil (2009a), the results show a decrease in developmental items of categories 1 and 2 and an increase of items in categories 3 and 4 in the sample of Yaqui children when compare with Maganto and Garaigordobil (2009a) standards. These differences indicate that the use of Maganto and Garaigordobil (2009a) scoring rules of the T2F-M is not adequate to establish interpretations of Yaqui children performance in this test because, in all age groups, the use of these norms underestimates their performance. In general terms, the Yaqui children of this sample drew less T2F-M elements in their drawings of feminine and masculine human figures, than did the Spanish children sample of Maganto and Garaigordobil (2009a). We conclude that T2F-M is useful in assessing the level of conceptual maturity of the indigenous community children, but only the use of local rules can ensure the correct interpretations of the scores obtained by these children.

Key words: Draw-a-Person Test; Yaqui indians; Conceptual maturity; Validity; Norms.

\section{INTRODUCCIÓN}

Los tests del dibujo de la figura humana como una medida de la habilidad intelectual han sido algunos de los instrumentos psicológicos más utilizados en la práctica clínica y educativa infantil y adolescente (Archer, Maruish, Imhof \& Piotrowski, 1991; Cashel, 2002; Costenbader, Rohrer \& Difonzo, 2000; Flanagan \& Motta, 2007). Este amplio uso se debe fundamentalmente a que la tarea que se propone es una actividad natural en la infancia, por lo que resulta atractiva y no amenazante para los niños, y a que su carácter no verbal y breve permite la evaluación de niños con problemas de lenguaje, hiperactividad, atención y aprendizaje (Abell, Wood \& Liebman, 2001; Scott, 1981) o que 
no hablan la lengua del país (Abell et al., 2001).

El primer sistema de puntuación estandarizado de esta técnica de evaluación apareció en 1926, con la publicación del Test del Dibujo de un Hombre de Goodenough (1926). Esta autora desarrolló una escala de ítemes que hacían referencia a la presencia o ausencia de diferentes partes del cuerpo humano y a las relaciones entre esas partes. Estos ítemes se obtuvieron empíricamente a partir de una muestra de niños de distintas edades. En la década de los años 60, Harris publicó una versión revisada y actualizada del Test de Goodenough (Harris, 1991). Su trabajo extendió el método de esta autora a la adolescencia y añadió al dibujo de un hombre, el dibujo de una mujer y el de sí mismo. En 1965 / 2002, Koppitz publica un sistema que permitía, a la vez, una evaluación madurativa conceptual de los niños y una evaluación proyectiva de los problemas emocionales, a partir del dibujo de una persona. El sistema para la evaluación madurativa conceptual de Koppitz (2002) consiste en una escala breve formada por 30 ítemes inspirados en el Test de Goodenough-Harris (Harris, 1991).

Los sistemas de Goodenough-Harris (Harris, 1991) y el de Koppitz (2002) han recibido críticas como medidas de funcionamiento intelectual, fundamentadas en los resultados de los estudios de validez de estos tests, que han encontrado índices de correlación entre bajos y moderados con otros tests de inteligencia y un bajo poder predictivo del rendimiento académico de los niños (Abell, Von Briesen \& Watz, 1996; Aikman, Belter \& Finch, 1992; Motta, Little \& Tobin, 1993; Scott, 1981; Ter Laak, de Goede, Aleva \& Van-Rijswijk, 2005). A pesar de estas críticas, el interés por el uso de esta técnica en la práctica de la evaluación psicológica continúa vigente, prueba de ello es que en los últimos años han surgido nuevos sistemas de puntuación, con el objetivo de actualizar y revisar las versiones de Goodenough-Harris y de Koppitz (Maganto \& Garaigordobil, 2009a; Naglieri, 1988; Reynolds \& Hickman, 2004; Sisto, 2006; Wechsler, 1998).
En Latinoamérica, el interés por la técnica del dibujo de la figura humana se manifiesta en las recientes publicaciones sobre estudios normativos y de validez de los diversos sistemas de puntuación del dibujo de la figura humana (Bandeira, Costa \& Arteche, 2008; Carreras, Uriel \& Fernández, 2013; FloresMendoza, Alves, Abad \& Lelé, 2010; Merino, Honores, García \& Salazar, 2007; Rosa, 2008; Rueda \& Sisto, 2006; Vélez et al., 2011). Los estudios normativos del sistema madurativo de Koppitz realizados en Perú (Merino et al., 2007) y Colombia (Vélez et al., 2011) encontraron un número mayor de ítemes evolutivos en los dibujos de los niños que participaron en estos estudios, en comparación al grupo de estandarización de Koppitz (2002). Según estos autores, estas diferencias se explican por el efecto Flynn (Flynn, 2007).

Los estudios realizados en Brasil sobre la validez de diversos sistemas del dibujo de la figura humana han mostrado en general, que las puntuaciones de los niños en estos sistemas correlacionan positivamente con otras medidas de inteligencia, aunque con valores que se sitúan entre bajos y moderados. Rosa (2008) estudió la correlación entre los sistemas Goodenough-Harris y Koppitz y el Test No Verbal de Inteligencia para Niños (Rosa \& Alves, 2000). Los resultados mostraron índices significativos y moderados (.606 y .575 , respectivamente). Con el sistema del dibujo de la figura humana de Wechsler (1998), Bandeira y colaboradores (2008) encontraron correlaciones significativas moderadas (.50) con el Test de Matrices Progresivas Coloreadas (Raven, 1993) y Flores-Mendoza y colaboradores (2010), índices de correlación significativos aunque bajos entre el funcionamiento de los niños en este sistema y el MPC (.204), la Escala Verbal del WISC-III (Wechsler, 1991) (.299), el Test No Verbal de Inteligencia para Niños (Rosa \& Alves, 2000) (.330) y el Test Gestáltico Visomotor de Bender (Koppitz, 1975) (-.396). Con el Dibujo de la Figura Humana - Escala Sisto (2006), Rueda y Sisto (2006) encontraron que este sistema diferencia las categorías de interpretación del MPC en las edades de 8, 9 y 10 años, pero no en el grupo de edad de 7 
años. En Argentina, Carreras y colaboradores (2013) encontraron índices de correlación significativos que oscilan de bajos (.179) a moderados (.474) entre el Test Gestáltico Visomotor de Bender (Koppitz, 1975) y la adaptación del sistema de Goodenough-Harris de Casullo (1988).

El Test del Dibujo de Dos Figuras Humanas (T2F - Maganto \& Garaigordobil, 2009a) surge de la necesidad de contar en España con un sistema de puntuación actualizado y revisado de los tests del dibujo de la figura humana. El objetivo del T2F es evaluar el nivel madurativo - mental y la problemática emocional en niños de 5 a 12 años de edad. La consigna es similar a la de la prueba de Machover (1949); en primer lugar se solicita el dibujo de una persona completa y a continuación, en otra hoja, el dibujo de una persona del sexo opuesto al dibujado.

Con respecto a la evaluación madurativomental, Maganto y Garaigordobil (2009a) especifican que el T2F-Madurativo (T2F-M) es una prueba sensible, más que específica, para medir esta capacidad. Permite una evaluación inicial (screening) para identificar niños con un posible retraso madurativo mental, pero no para establecer un diagnóstico de su nivel intelectual.

El proceso de construcción del T2F-M consistió en una selección preliminar de 60 ítemes obtenidos de una revisión de investigaciones anteriores sobre los tests del dibujo de la figura humana y de las propias experiencias profesionales de las autoras. Para aceptar estos ítemes en el test, las autoras determinaron que tenían que cumplir dos criterios: seguir un patrón evolutivo y correlacionar con el Test de Matrices Progresivas (TMPRaven, 2001), en una muestra normativa formada por 1.122 alumnos españoles de 5 a 12 años de edad. Los resultados de este estudio indicaron que 8 ítemes no cumplían con estos dos criterios y se eliminaron, y en el dibujo de figura femenina se eliminaron 3 ítemes más porque presentaban dificultades en la corrección. Finalmente, se consideraron 52 ítemes madurativos en el dibujo de la figura masculina y 49 en la figura femenina. Maganto y Garaigordobil (2009a) asignaron puntuacio- nes estandarizadas a los ítemes de cada figura humana y en cada grupo de edad, basándose en la frecuencia que presentaban en los dibujos de la muestra normativa. De este modo, establecieron cuatro categorías: (a) ítemes con una frecuencia de aparición de $85 \%$ o más, cuya puntuación estandarizada es 1 punto si están presentes y -1 punto si se omiten; (b) ítemes con una frecuencia entre el 84 y el 50\%, cuya puntuación es 2 puntos si están presentes y 0 punto si se omiten; (c) ítemes con una frecuencia entre el 49 y el $15 \%$, con una puntuación de 3 puntos si se dibujan y de 0 punto si se omiten y (d) los ítemes con una frecuencia de $14 \%$ o menos, que reciben 4 puntos si están presentes y 0 si están ausentes. Maganto y Garaigordobil (2009a) ofrecen baremos (puntuación percentil) para la interpretación de las puntuaciones directas totales, según el curso y sexo de los niños.

Con respecto a las propiedades psicométricas del T2F-M, Maganto y Garaigordobil (2009a) encuentran un nivel alto de consistencia interna $(\alpha=.74$ en la figura masculina, $\alpha=.89$ en la figura femenina y $\alpha=.86$ en ambas figuras). Las autoras estudiaron la validez a partir de las correlaciones y análisis de contingencia entre las puntuaciones del grupo normativo en el T2F-M y las puntuaciones en el TMP de Raven. Los resultados muestran índices de correlación significativos y positivos, con valores que oscilan de bajos (.39) a moderados (.69) entre el T2F$\mathrm{M}$ y el TMP (Maganto \& Garaigordobil, 2009 b). Por otro lado, el análisis de contingencia indicó que una ejecución baja en el T2F-M corresponde con alta probabilidad a una ejecución baja o inferior en el TMP, pero que una ejecución buena en el T2F-M no corresponde siempre a un nivel de inteligencia alta o superior en el TMP, por lo tanto, la capacidad predictiva negativa del T2F-M es mayor que la capacidad predictiva positiva (Maganto \& Garaigordobil, 2009a).

El carácter fundamentalmente no verbal de los tests del dibujo de la figura humana generó desde su inicio una gran expectativa por su aplicación en la evaluación multicultural. Sin embargo, los primeros estudios que compararon la ejecución de los niños de distintas 
culturas mostraron que la prueba dependía más de las diferencias en los antecedentes culturales, de lo que se había supuesto originalmente (Anastasi \& Urbina, 1998, Harris, 1991). La mayoría de los estudios transculturales realizados con el Test de Goodenough Harris y el sistema Koppitz han encontrado diferencias en las puntuaciones medias de los niños y en la frecuencia de determinados ítemes evolutivos (Dennis, 1966; Gonzales, 1982; Laosa, Swartz \& Díaz Guerrero, 1974; Mehryar, Tashakkori, Yousefi \& Khajavi, 1987; Vedder, Van de Vijfeijken \& Kook, 2000).

Algunos estudios han encontrado una frecuencia más baja de ítemes en el test de Goodenough-Harris y el sistema Koppitz en niños de culturas distintas a la estadounidense y europea (Hutz \& Antoniazzi, 1995; Laosa et al., 1974; Mehryar, Tashakkori, Yousefi \& Khajavi, 1987; Vedder et al., 2000). Por ejemplo, Laosa y colaboradores (1974) encontraron puntuaciones medias más bajas en el Test de Goodenough-Harris en una muestra de niños mexicanos, que en una muestra de niños anglo-americanos de igual edad, sexo y nivel socioeconómico, que el grupo de niños mexicanos. El estudio de Mehryar y colaboradores (1987) realizado con un grupo de niños iraníes, también mostró puntuaciones medias en el test de Goodenough-Harris más bajas en relación a las de las normas estadounidenses de este test. Con el sistema de Koppitz, Vedder y colaboradores (2000) encontraron puntuaciones más bajas en los niños de Curacão, en comparación con las normas holandesas de Van de Vijfeijken (1994, citado en Vedder et al., 2000) y las estadounidenses de Koppitz (2002). Hutz y Antoniazzi (1995) encontraron en la mayoría de los grupos de edad de una muestra de niños brasileños una disminución de los ítemes esperados y un aumento de los excepcionales en relación a las normas de Koppitz.

El nivel socioeconómico es una variable que también afecta el rendimiento de los niños en los tests del dibujo de la figura humana. En general, se ha encontrado una mejor ejecución en niños de niveles socioeconómi- cos medios y altos, que en los niños de bajos recursos socioeconómicos (Mehryar et al., 1987; Ozer, 2009; Pfeffer \& Oluwu, 1986). La revisión de la literatura sobre el test Goodenough-Harris realizada por Scott (1981) mostró que el nivel socioeconómico es una de las variables que más influye en el rendimiento de los niños en estos tests.

La influencia de los elementos socioculturales en la ejecución de los niños en los tests del dibujo de la figura humana confirma la necesidad de contar con normas locales para una interpretación más válida y justa de las puntuaciones. Las normas de Standards for Educational and Psychological Testing (American Educational Research Association -AERA-, APA y el National Council on Measurement in Education-NCME-, 1999) establecen que se debe obtener la validez de las puntuaciones de los tests independientemente para cualquier población con diferencias culturales (etnia, o nivel socioeconómico) con respecto a la población para la que se construyó el test. Precisamente, sobre estos tests, autores como Harris (1991) y Cox (1993) han expresado que para obtener resultados más válidos, la prueba se debería reestandarizar para cada grupo con características culturales y sociales diferentes.

En México, el sistema de Koppitz (2002) es el más utilizado (Esquivel, Heredia \& Lucio, 2007); en concreto, en la comunidad indígena yaqui es el sistema que utilizan los psicólogos que trabajan con niños y adolescentes. Sin embargo, este sistema es anticuado puesto que se publicó hace más de 40 años. Además existen ciertas imprecisiones de la traducción al español de esta prueba (González, 1988) y una ambigüedad de los criterios de corrección de determinados ítemes (Vélez et al., 2011). Estas limitaciones justifican el cambio a un sistema actualizado y revisado de las pruebas del dibujo de la figura humana, como el T2F (Maganto \& Garaigordobil, 2009a). Sin embargo, este test está construido y estandarizado en una muestra de niños españoles, por lo que, si se pretende utilizar con los niños yaquis, es necesario estudiar sus cualidades psicométricas en esta población y obtener normas locales para 
una interpretación más válida y justa de la ejecución de estos niños. Por tal motivo para realizar el estudio que se informa se plantearon los siguientes objetivos:

1.- Analizar la validez y fiabilidad del T2F - Madurativo en una muestra de niños yaquis de 5 a 11 años y11 meses de edad, examinando la validez a través del estudio evolutivo de los ítemes madurativos y de la correlación entre el funcionamiento de los niños en el T2F-M y la prueba de inteligencia Test de Matrices Progresivas Coloreadas (Raven, 1993). En cuanto a la fiabilidad, estudiar la consistencia interna de los ítemes evolutivos y el acuerdo interevaluadores.

2.- Obtener normas específicas en la población infantil de este grupo cultural y compararlas con las de Maganto y Garaigordobil (2009a), con el fin de determinar la adecuación de estas normas en la población infantil yaqui.

De acuerdo con los estudios anteriores que han encontrado diferencias en el funcionamiento de niños de culturas distintas a la estadounidense y europea en los tests del dibujo de la figura humana de Goodenough-Harris y el sistema Koppitz (Dennis, 1966; Hutz \& Antoniazzi, 1995; Laosa et al., 1974; Mehryar et al., 1987; Vedder et al., 2000) y de nivel socioeconómico bajo (Mehryar et al., 1987; Ozer, 2009), se conjetura que existirán diferencias en las normas que se identifiquen en la muestra de niños yaquis comparadas con las de Maganto y Garaigordobil (2009a).

\section{CONTEXTO DE LA POBLACIÓN}

El grupo indígena yaqui o yoremes, como ellos se denominan, pertenece a la familia Yuto-Azteca, grupo Pima y subgrupo Opata Cahita Tarahumara (Fábila, 1978). Antes de la conquista española, ellos vivían en pequeños agrupamientos en los márgenes del curso inferior del río Yaqui, en el actual estado de Sonora. Eran agricultores seminómadas y guerreros (Spicer, 1994). Con la colonización de los misioneros jesuitas, la población yaqui se concentró en ocho pueblos, que actualmente constituyen las unidades fundamentales de organización política y social de este grupo. El número de habitantes yaquis en el año 2000 se situaba alrededor de 34.877 , distribuidos en 52 comunidades y principalmente en los ocho pueblos tradicionales (Martínez, 2010). La lengua yaqui pertenece a la familia lingüística Yuto-Nahua y actualmente es hablada por 14.162 habitantes (Diario Oficial, 2010).

El grado de marginación de las comunidades yaquis es alto (Consejo Nacional de Población -CONAPO-, 2010). La mayoría de los yaquis trabajan como mano de obra barata en tareas agrícolas en el campo, la construcción, las maquiladoras y en el sector pesquero durante la temporada alta del camarón. Las mujeres yaquis que trabajan fuera del hogar, lo hacen como dependientas en tiendas y como trabajadoras domésticas (Moctezuma, 2007).

En el territorio yaqui se ofrece educación escolar desde el nivel inicial hasta el medio superior. Los programas de estudio se basan en los programas nacionales establecidos por la Secretaría de Educación Pública (SEP) y utilizan los libros de texto en español de la SEP y los libros en yaqui "Jiak Noki", elaborados por el Proyecto Educativo de la Tribu Yaqui.

\section{MÉTOdO}

Para la obtención de los datos y su análisis se utilizó una metodología cuantitativa con alcance correlacional y un diseño evolutivo transversal.

\section{PARTICIPANTES}

La muestra estuvo formada por 654 alumnos (48\% varones y 52\% mujeres) de 8 escuelas públicas situadas en las comunidades yaquis (Sonora, México), de tercer nivel de preescolar a sexto grado de primaria. El ta- 
maño de la muestra es representativo de la población total de alumnos de estos niveles escolares (en el ciclo escolar 2011 - 2012, esta población consistía aproximadamente en 6.007 alumnos - Secretaría de Educación y Cultura, 2012). La muestra es no aleatoria, porque el personal de la Unidad de Servicio de Apoyo a la Educación Regular (USAER) de la zona se encargó de indicar los centros escolares que estaban dispuestos a colaborar en la investigación.

Se excluyeron de la muestra los niños que no tenían entre 5 y 11 años y 11 meses de edad, niños que presentaban deficiencias visuales y auditivas que no podían corregirse adecuadamente con el uso de lentes y audífonos, respectivamente, y los que no siguieron las instrucciones adecuadamente. En la Tabla 1 se presenta la distribución de los participantes por edad.

\section{INSTRUMENTOS}

Se utilizaron los siguientes instrumentos: la Escala Madurativa del Test de dos Figuras Humanas (T2F-M - Maganto \& Garaigordobil, 2009a) y la forma cuadernillo del Test de Matrices Progresivas Coloreadas (MPC Raven, 1993). El MPC evalúa la habilidad de razonamiento abstracto o no verbal. Este test está formado por ilustraciones de figuras geométricas abstractas con fondos coloreados, que representan un patrón de pensamiento, una matriz, de manera incompleta. La tarea del sujeto consiste en elegir la figura que falta entre las alternativas proporcionadas.

\section{Procedimiento}

Se solicitó al psicólogo de la USAER de la zona escolar yaqui su apoyo para la realización de esta investigación. El psicólogo se encargó de comunicar a maestros, alumnos y padres de distintos centros escolares, los objetivos de la investigación, en qué consistiría la participación de los alumnos y el uso confidencial de los resultados individuales de la prueba. Finalmente, el psicólogo indicó los 8 centros escolares que aceptaron participar. El estudio cumplió con las normas éticas para la investigación con seres humanos de la APA (autorización institucional y consentimiento informado).

Tres psicólogos aplicaron el T2F y el MPC individualmente a todos los alumnos que cumplían con los criterios de selección de la muestra. Las administraciones se realizaron en una única sesión, en primer lugar se aplicó el T2F y luego el MPC, según las instrucciones de los manuales respectivos, en salas confortables de los centros escolares, dentro del horario escolar y durante los meses de agosto a diciembre del año 2012. Dos de los psicólogos aplicaron las pruebas en idioma español y uno en yaqui o español, dependiendo de la lengua materna de los alumnos.

\section{ANÁLISIS DE LOS DATOS}

Se seleccionaron al azar 31 protocolos del T2F-M, que fueron calificados por tres psicólogos siguiendo los criterios de corrección de Maganto y Garaigordobil (2009a). Se analizó el acuerdo intercalificadores del número total de ítemes presentes en estos 31 protocolos y se obtuvo un coeficiente de correlación intraclase promedio de $.993(p=.000)$, lo que indica un acuerdo intercalificadores muy alto, por lo tanto el resto de los protocolos se dividió entre los tres evaluadores y cada uno fue calificado una sola vez. Los MPC se calificaron siguiendo el criterio del manual de esta prueba. Las puntuaciones de ambos tests se ingresaron en el programa IBM SPSS Statistics 19.0.

\section{Resultados}

Para examinar si existía un patrón de desarrollo de los ítemes madurativos del T2F-M (Maganto \& Garaigordobil, 2009a) se realizó un análisis de variancia de un factor, que mostró una diferencia significativa en el número total de ítemes presentes en ambos dibujos de los distintos grupos de edad $[F(6$, $654)=100.374, p<.01]$. En la Tabla 1 se ob- 
serva cómo la media de ítemes presentes en los dos dibujos aumenta a medida que se incrementa la edad de los participantes, de 27.52 en el grupo de 5 años a 59.20 a los 11 años de edad.

Los resultados del análisis de variancia de un factor realizados con el número total de ítemes presentes en los dibujos de la figura masculina, por un lado, y en el de la femenina, por otro, también mostraron diferencias significativas según la edad de los participantes $[F(6,654)=93.306, p<.01 \mathrm{y}$ $F(6,654)=85.475, p<.01]$, respectivamente.

El método post hoc de Bonferroni indicó que la mayoría de las comparaciones de las puntuaciones medias del total de ítemes presentes en los dos dibujos de los distintos grupos de edad difieren significativamente entre sí $(p<.05)$, excepto las comparaciones entre los grupos de 7 y 8 años $(p=1.000), 8$ y 9 años $(p=.165), 9$ y 10 años $(p=1.000)$ y 10 y 11 años $(p=1.000)$. Un contraste polinómico entre las puntuaciones medias de ítemes presentes en los T2F-M y los distintos grupos de edad mostró que entre ambas variables existe una relación lineal significativa $[F(1$, $654)=558.166, p<.01]$, es decir, que se produce un aumento de la media de ítemes presentes en los dibujos de ambas figuras a medida que se incrementa la edad de los niños de la muestra.

Un análisis de variancia univariante (sexo $\mathrm{x}$ edad) mostró que no existe diferencia significativa en las puntuaciones medias del total de ítemes presentes en los dos dibujos según el sexo de los participantes $[F(1$, $654)=2.824, p>.05]$, ni en los efectos de la interacción de las variables sexo y grupos de edad $[F(6,654)=1.119, p>.05]$.

Con el fin de obtener información sobre la validez concurrente de las puntuaciones del T2F-M, se correlacionó el número total de ítemes presentes en los dos dibujos con la puntuación directa de los participantes en el MPC. Los resultados obtenidos en ambas figuras (ver Tabla 2) indican que en la mayoría de los grupos de edad la correlación fue significativa, positiva y con valores bajos (entre .211 y .382), a excepción del grupo de
7 años, donde el índice de correlación fue no significativo. Considerando la muestra total de niños, la correlación fue positiva, significativa y presentó un valor moderado (.608). En el dibujo de la figura masculina, los índices de correlación obtenidos entre el número total de ítemes presentes y las puntuaciones en el MPC son, en todos los grupos de edad, significativos, positivos y con valores bajos (entre .238 y .352). En el dibujo de la figura femenina existen tres grupos $(7,8$ y 11 años de edad) donde no se encontraron correlaciones significativas entre el número de ítemes dibujados y el MPC; en el resto de los grupos de edad los índices de correlación fueron significativos, positivos y con valores bajos (entre .249 y .385).

El análisis de la consistencia interna de los ítemes del T2F-M en esta población, se obtuvo a partir del modelo Alpha de Cronbach y del método de dos mitades (ver Tabla 3). En los dibujos de ambas figuras, los coeficientes Alpha en las distintas edades oscilan entre valores altos (.891) y muy altos (.922) y en la muestra total, el coeficiente fue muy alto (.950). Con el método de dos mitades, los coeficientes Spearman-Brown en los distintos grupos de edad se situaron también entre altos (.814) y muy altos (.921) y en la muestra total fue muy alto (.939). En el dibujo de la figura masculina, los coeficientes Alpha en los distintos grupos de edad fueron altos (entre .798 y .865) y en la muestra total fue muy alto (.908), mientras que los coeficientes Spearman-Brown en los distintos grupos de edad fueron moderados (entre .412 y .688) $\mathrm{y}$ en la muestra total, alto (.769). En el dibujo de la figura femenina se obtuvieron coeficientes Alpha altos en las distintas edades (entre .802 y.869) y en la muestra total muy alto (.905), por otro lado, los coeficientes Spearman-Brown en los distintos grupos de edad se situaron entre moderados $(.503)$ y altos (.792) y en la muestra total, alto (.801).

Para obtener las normas del T2F-M de la muestra de niños yaquis, se aplicó el criterio de asignación de puntuaciones estandarizadas de Maganto y Garaigordobil (2009a) a los datos obtenidos en este estudio en cada ítem madurativo de ambas figuras y en los distin- 
tos grupos de edad. Es decir, los ítemes con una frecuencia de aparición de $85 \%$ o más se clasificaron en la categoría 1 (si están presentes se les otorga 1 punto y si se omiten se penaliza restándoles 1 punto), los ítemes con una frecuencia entre el 84 y el 50\% se clasificaron en la categoría 2 (si están presentes se les otorga 2 puntos y si están ausentes, 0 punto), los ítemes con una frecuencia entre el 49 y $15 \%$ se clasificaron en la categoría 3 (si están presentes se les otorga 3 puntos y si están ausentes 0 punto) y los ítemes con una frecuencia de $14 \%$ o menos se clasificaron en la categoría 4 (si están presentes reciben 4 puntos y si están ausentes 0 punto). En los casos que presentaban diferencias en la clasificación de los ítemes con respecto a las normas de Maganto y Garaigordobil (2009a), se estudió la bondad de ajuste de las frecuencias obtenidas a las distintas proporciones del criterio de asignación de puntuaciones, con la prueba $j i$ cuadrado para una muestra. Por ejemplo, en el caso de los ítemes que están clasificados en la categoría 1 en las normas de Maganto y Garaigordobil (2009a), pero que no presentaban el porcentaje suficiente para ser considerados dentro de esta categoría en los niños yaquis, se estudió la bondad de ajuste de las frecuencias obtenidas a la proporción 85; en los ítemes de la categoría 2 con las proporciones 84 ó 50 (según el valor de las frecuencias obtenidas se acerca más a una u otra proporción); en los ítemes de la categoría 3 con la proporción 49 ó 15 (según el valor de la frecuencia se acerca más a uno u otro valor) y los ítemes de la categoría 4 con la proporción 14. Cuando la diferencia con respecto a la frecuencia observada no era significativa $(p>.05)$ se mantuvo la categoría del ítem de las normas de Maganto y Garaigordobil (2009a); en cambio cuando la diferencia con respecto a la frecuencia esperada era significativa $(p<.05)$, se modificó la categoría del ítem, clasificándose según el criterio de asignación de puntuaciones de estas autoras. En las Tablas 4 y 5 se presentan los resultados de este análisis.

Los resultados indican diferencias entre las normas de puntuación de los dibujos de la figura masculina y femenina del estudio de Maganto y Garaigordobil (2009a) y las iden- tificadas en la muestra de niños yaquis (ver Tablas 4 y 5). Básicamente estas diferencias consisten en una disminución de los ítemes de las categorías 1 y 2 y en un aumento del número en las categorías 3 y 4 en la muestra de niños yaquis, en comparación con las normas de Maganto y Garaigordobil (2009a).

En la Tabla 6 se presentan los valores percentiles para ambos sexos que se han calculado de las puntuaciones directas totales en el T2F-M, obtenidas de las normas de puntuación de la muestra de niños yaquis.

\section{Discusión}

Los resultados sobre las cualidades psicométricas (validez y confiabilidad) del T2F-M analizados en este estudio, indican que es un test adecuado para evaluar el nivel madurativo conceptual en niños de la comunidad yaqui.

El primer aspecto analizado sobre la validez fue el análisis de las diferencias evolutivas de los ítemes del test e indicó diferencias estadísticamente significativas en el número total de ítemes madurativos dibujados según las edad de los niños, con un aumento en la media de los ítemes dibujados en ambas figuras a medida que se incrementa la edad de los niños de la muestra. Este incremento de los ítemes dibujados en el T2F-M confirma la relación con la edad y maduración de los ítemes en una población distinta para la que se construyó el test.

El segundo aspecto, que tiene que ver con la validez concurrente del T2F-M, mostró que existe una asociación de baja a moderada entre el número de detalles que dibujan los niños en el T2F-M y la habilidad de razonamiento abstracto o no verbal que evalúa el MPC. Este resultado coincide con el estudio de Maganto y Garaigordobil (2009b), en el que encontraron índices de correlación significativos con valores que se sitúan de bajos a moderados entre el T2F-M y el TMP. También es consistente con los resultados de los estudios de validez realizados con otros sistemas de puntuación de la figura humana (Bandeira et al., 
2008; Flores-Mendoza et al., 2010; Rosa, 2008).

En cuanto a la fiabilidad, los coeficientes Alpha de Cronbach y de Spearman-Brown obtenidos para el total de la muestra indican que existe una consistencia interna muy alta entre los ítemes evolutivos del T2F-M en esta población. Maganto y Garaigordobil (2009a) también encontraron un nivel alto de consistencia interna en el T2F-M. Por otro lado, los resultados del acuerdo interevaluadores muestran una coincidencia muy alta entre las puntuaciones otorgadas por los distintos evaluadores de los protocolos del T2F-M, lo cual es congruente con los resultados obtenidos en estudios anteriores realizados con otros sistemas del dibujo de la figura humana, que han analizado este tipo de fiabilidad (Abell et al., 1996; Gayton, Tavormina, Evans \& Schuh, 1974; Gonzales, 1982; Hutz \& Antoniazzi, 1995; Merino et al., 2007; Ozer, 2009; Vélez et al., 2011).

El análisis de las diferencias de sexo en el número de ítemes dibujados en el T2F-M, indicó que no existen diferencias significativas en función de esta variable. Este resultado es consistente con los obtenidos en algunos estudios realizados con el sistema Koppitz y el Test de Goodenough-Harris (Hutz \& Antoniazzi, 1995; Jegede \& Bamgboye, 1982) y con los datos proporcionados por Harris (1991) sobre el funcionamiento similar en niños y niñas de varios grupos de indígenas norteamericanos. Sin embargo, difiere de los resultados obtenidos por Maganto y Garaigordobil (2009a) en la muestra de niños españoles y los de Koppitz (2002), que mostraron una ejecución superior en las mujeres con respecto a los varones.

En cuanto al segundo objetivo propuesto, la obtención de normas específicas en el T2F$\mathrm{M}$ y su comparación con las de Maganto y Garaigordobil (2009a) para determinar su adecuación en la población yaqui, los resultados muestran diferencias, que, en general, consisten en una disminución de los ítemes de las categorías 1 y 2 y un aumento de los ítemes de las categorías 3 y 4 en la muestra de niños yaquis, en relación con las normas de Maganto y Garaigordobil (2009a). En cuanto a los ítemes de las categorías 1 y 2 , se encontró una disminución de éstos en todos los grupos de los niños yaquis, tanto en el dibujo de la figura masculina como en el de la femenina, a excepción del grupo de 6 años, que en el dibujo de la figura masculina mantuvo el número de ítemes de la categoría 2 en relación a las normas de Maganto y Garaigordobil (2009a). Con respecto a los ítemes de las categorías 3 y 4, existe un aumento en todos los grupos de la muestra de niños yaquis en relación con las normas españolas, a excepción de los grupos de niños de menor edad (5 y 6 años), que obtuvieron un número inferior o semejante de ítemes de la categoría 4, en comparación con las normas de Maganto y Garaigordobil (2009a).

Los ítemes que han presentado más diferencias, tanto en el dibujo de la figura masculina como en el de la femenina, son el 13 "nariz: orificios o fosas nasales", el 14 "nariz: estructura completa", el 17 "boca: dos labios", el 22 "cara: mofletes" y el 29 "tronco: cintura" (este último sólo en el dibujo de la figura femenina), que en todos los grupos de edad de la muestra de niños yaquis se han clasificado en la categoría 4, es decir, han presentado un porcentaje de aparición en los dibujos inferior al 14\%, a diferencia de la muestra de Maganto y Garaigordobil (2009a) en la que el dibujo de estos ítemes ha superado este porcentaje en los distintos grupos de edad y se ha clasificado en las categorías 2 ó 3.

Estas diferencias indican que la utilización de las normas de puntuación del T2F-M de Maganto y Garaigordobil (2009a) no es adecuada para establecer interpretaciones del funcionamiento de los niños yaquis en este test porque, en todos los grupos de edad, el uso de estas puntuaciones subestima su funcionamiento. En general, los niños yaquis de esta muestra dibujaron menos elementos del T2F-M en sus dibujos de la figura humana masculina y femenina, que la muestra de niños españoles. Algunos estudios transculturales realizados con el Test de Goodenough Harris y el sistema de Koppitz también han encontrado una frecuencia más baja de ítemes madurativos en niños de culturas distintas a la estadounidense y europea (Hutz \& 
Antoniazzi, 1995; Laosa et al., 1974; Mehryar et al., 1987). Las explicaciones dadas sobre estas diferencias en el funcionamiento de los niños apuntan a la influencia de elementos culturales y socioeconómicos. Diversos autores han mencionado que la importancia que otorga un grupo cultural a la expresión artística puede influir en la ejecución de los niños en estos tests (Dennis, 1966; Mehryar et al., 1987). En el grupo indígena yaqui, el desarrollo artístico es relativamente escaso, comparado con los pueblos de Mesoamérica y con el mundo occidental, por lo que los niños yaquis pueden tener menos experiencia con actividades artísticas y esto se refleja en sus dibujos de la figura humana. En relación al nivel socioeconómico, la mayoría de los estudios han encontrado una mejor ejecución en el Test de Goodenough-Harris y el sistema Koppitz de niños de niveles socioeconómicos medios y altos, que de bajos recursos (Mehryar et al., 1987; Pfeffer \& Oluwu, 1986; Ozer, 2009; Scott, 1981). Sobre este aspecto, hay que señalar el alto grado de marginación de las comunidades yaquis, la mayoría de su población vive en zonas rurales, no disponen de los servicios sociales básicos, con bajos ingresos económicos, vivienda inadecuada y baja escolaridad (CONAPO, 2010). Estas condiciones socioeconómicas $\mathrm{y}$, fundamentalmente, las pocas oportunidades educativas que tienen estos niños posiblemente influyen en las diferencias en el funcionamiento que han pre- sentado los niños yaquis de este estudio en el T2F-M, en comparación con el grupo normativo de Maganto y Garaigordobil (2009a). Autores como Martlew y Connolly (1996) y Cox (1993) han señalado la importancia de la escolarización en la habilidad para el dibujo.

Entre las limitaciones de esta investigación se encuentra que el número de participantes por cada grupo de edad no es representativo de la población de alumnos de los niveles escolares correspondientes a estas edades, lo cual genera error de muestreo al analizar los datos por edades.

Por otro lado, se sugiere para futuras investigaciones obtener información sobre el estilo de crianza de los padres yaquis y las condiciones de salud y nutrición infantil, aspectos que pueden influir en el funcionamiento de estos niños en el T2F-M.

En conclusión, los resultados de esta investigación indican que el T2F-M es útil para evaluar el nivel de madurez conceptual de los niños de la comunidad indígena yaqui, pero con el uso de normas locales que aseguren la realización de inferencias e interpretaciones correctas sobre el significado de las puntuaciones que obtienen los niños. Dentro de una batería de tests que incluya otras medidas de funcionamiento intelectual, la utilización de estas normas del T2F-M puede ayudar a los psicólogos que trabajan con esta población infantil a tomar decisiones más adecuadas sobre las intervenciones psicoeducativas que necesitan los niños evaluados. 
TABLA 1

MEDIAS Y DESVIACIONES TIPO DEL TOTAL DE ÍTEMES PRESENTES EN EL T2F-M SEGÚN GRUPOS DE EDAD

\begin{tabular}{|l|c|cc|cc|cc|cc|}
\hline $\begin{array}{c}\text { Grupos } \\
\text { de } \\
\text { edad }\end{array}$ & $n$ & \multicolumn{2}{|c|}{ Figura masculina } & \multicolumn{2}{|c|}{ Figura femenina } & \multicolumn{2}{|c|}{$\begin{array}{l}\text { Figuras masculina y } \\
\text { femenina }\end{array}$} & Mínimo & Máximo \\
\hline 5 años & 105 & $M$ & $D T$ & $M$ & $D T$ & $M$ & $D T$ & & \\
6 años & 99 & 13.72 & 5.943 & 13.80 & 6.404 & 27.52 & 11.789 & 5 & 66 \\
7 años & 103 & 23.12 & 5.460 & 17.90 & 5.900 & 35.21 & 11.852 & 7 & 68 \\
8 años & 91 & 24.86 & 6.008 & 24.08 & 5.569 & 46.19 & 10.480 & 16 & 72 \\
9 años & 100 & 27.03 & 6.108 & 26.61 & 5.715 & 53.64 & 11.327 & 23 & 75 \\
10 años & 82 & 28.16 & 5.516 & 27.66 & 5.627 & 55.82 & 10.541 & 34 & 84 \\
11 años & 74 & 30.18 & 5.964 & 29.03 & 6.084 & 59.20 & 11.235 & 31 & 89 \\
\hline Total & 654 & 23 & 8.160 & 22.79 & 7.763 & 45.79 & 15.434 & 5 & 89 \\
\hline
\end{tabular}

TABLA 2

Coeficientes de correlación de PeARson entre los ítemes PRESEntes en el T2F-M y La Puntuación DIRECTA EN EL MPC

\begin{tabular}{|l|c|c|c|c|}
\hline Grupos de edad & $n$ & Figura masculina & Figura femenina & Figuras masculina y femenina \\
\hline 5 años & 105 & $.343^{* *}$ & $.385^{* *}$ & $.382^{* *}$ \\
6 años & 99 & $.314^{\star *}$ & $.308^{* *}$ & $.324^{* *}$ \\
7 años & 103 & $.238^{*}$ & $.103^{a}$ & $.189^{a}$ \\
8 años & 91 & $.257^{*}$ & $.131^{a}$ & $.211^{*}$ \\
9 años & 100 & $.352^{\star *}$ & $.372^{* *}$ & $.377^{* *}$ \\
10 años & 82 & $.266^{*}$ & $.249^{*}$ & $.272^{*}$ \\
11 años & 74 & $.252^{*}$ & $.215^{a}$ & $.250^{*}$ \\
\hline Total & 654 & $.603^{* *}$ & $.576^{* *}$ & $.608^{* *}$ \\
\hline
\end{tabular}

a $p>.05$

*La correlación es significativa al nivel .05 (bilateral)

**La correlación es significativa al nivel .01 (bilateral) 


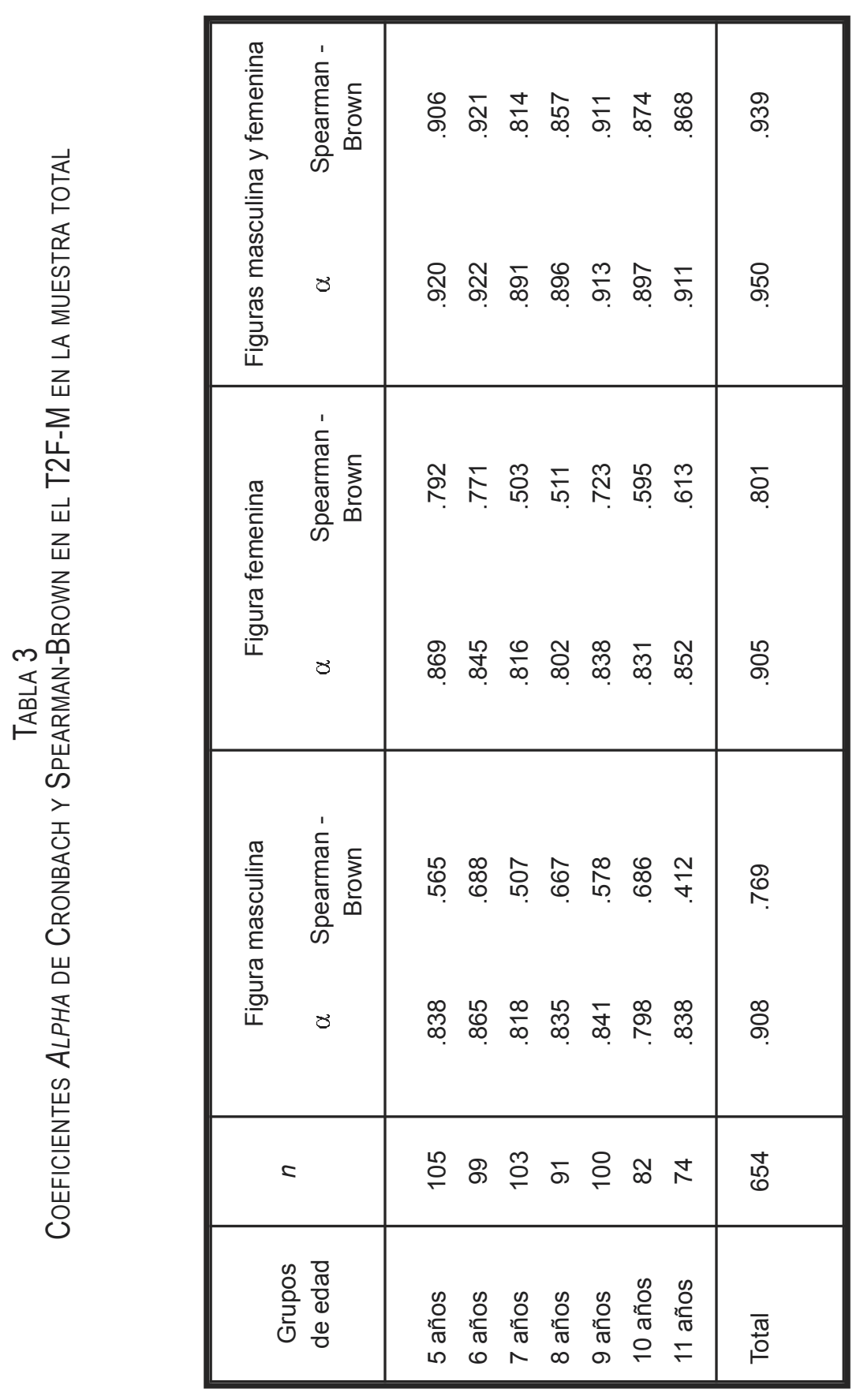




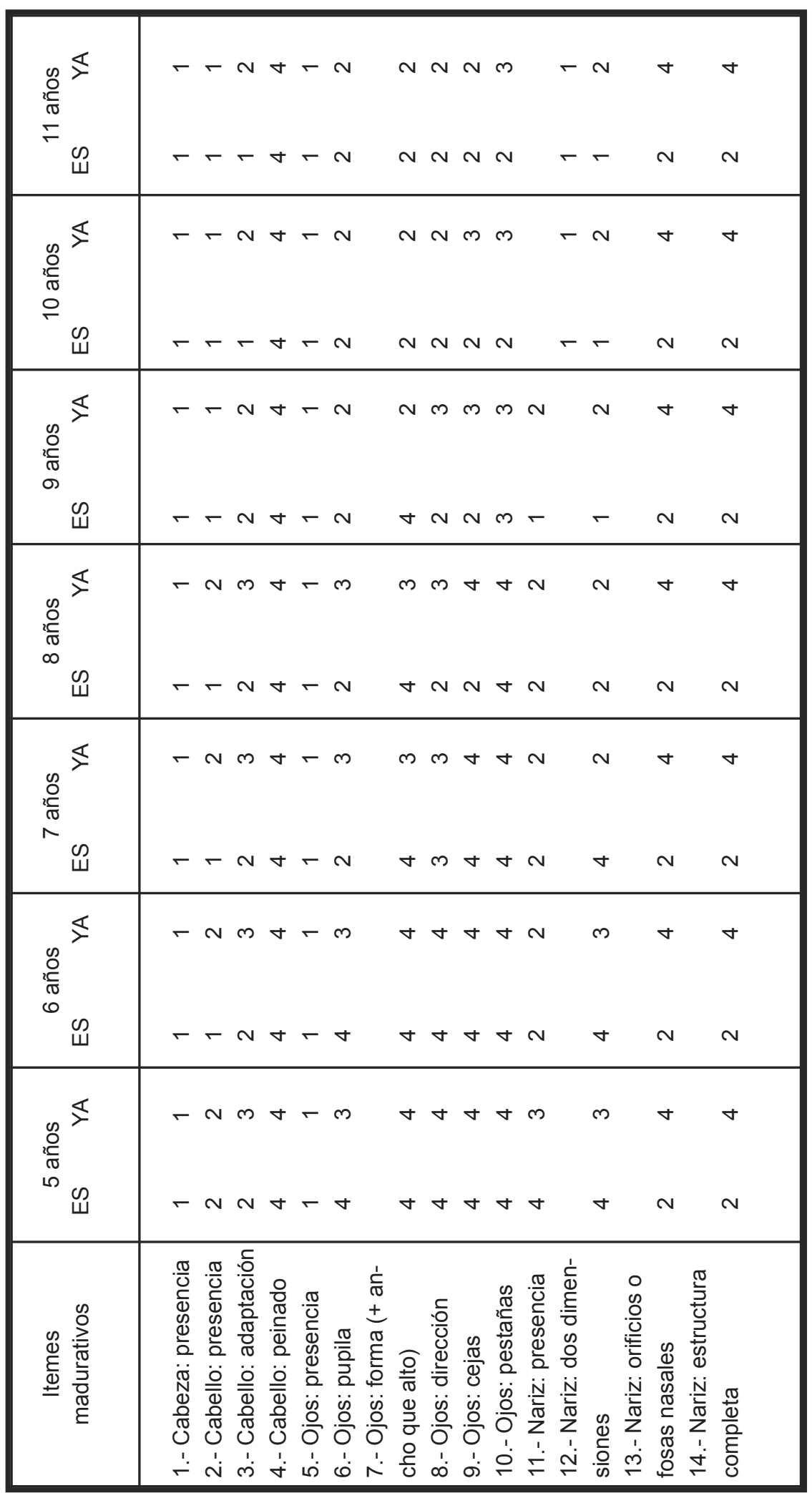




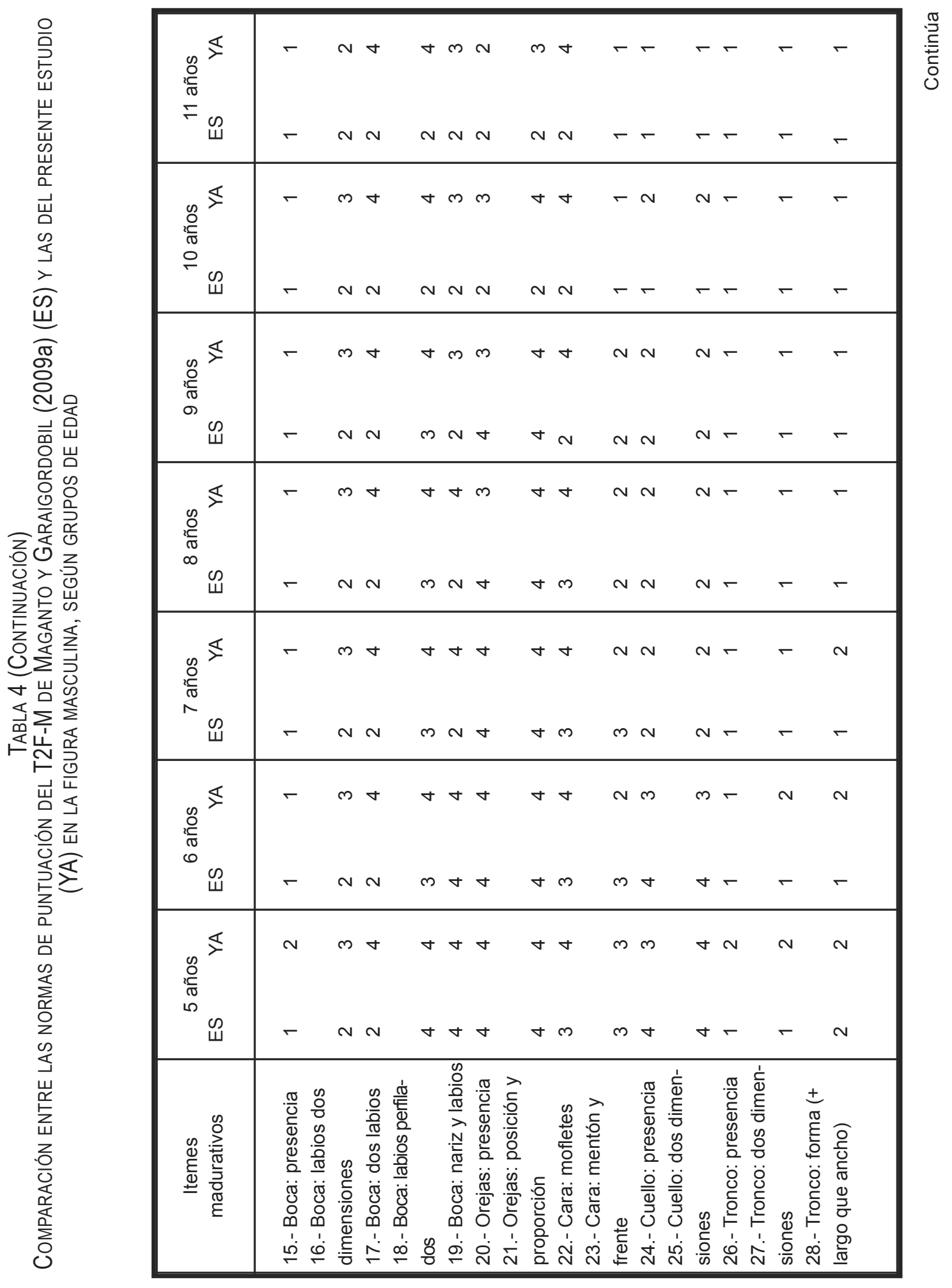




\begin{tabular}{|c|c|c|c|c|c|c|c|c|c|}
\hline 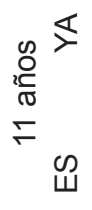 & $\begin{array}{l}n+ \\
+m\end{array}$ & $r$ & $\begin{array}{l}- \\
-\end{array}$ & $\begin{array}{l}N+N \\
-N N\end{array}$ & $\begin{array}{l}- \\
-\end{array}$ & $\begin{array}{l}N-c \\
r-r\end{array}$ & & $\begin{array}{l}m \\
N\end{array}$ & $\begin{array}{l}m \\
N\end{array}$ \\
\hline 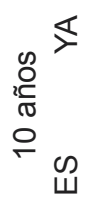 & $\begin{array}{l}n+ \\
\sim m\end{array}$ & $r$ & - & $\begin{array}{l}n+m \\
\sim N N\end{array}$ & - & $\begin{array}{l}N \sim C \\
r-r\end{array}$ & - & $\begin{array}{l}m \\
N\end{array}$ & $\begin{array}{l}m \\
N\end{array}$ \\
\hline 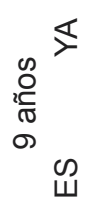 & $\begin{array}{l}A \\
A+\end{array}$ & - & - & $\begin{array}{l}n+m \\
-N N\end{array}$ & - & $\begin{array}{l}m \sim c \\
-r\end{array}$ & - & $\sim$ & $\begin{array}{c}2 \\
\theta \\
\theta\end{array}$ \\
\hline 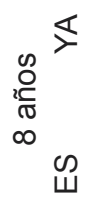 & $\begin{array}{l}A \\
A+\end{array}$ & - & - & $\begin{array}{l}N+m \\
\sim \sim N\end{array}$ & - & $\begin{array}{l}m \sim c \\
n-.\end{array}$ & - & $\forall$ & $\begin{array}{l}\checkmark \\
\nabla\end{array}$ \\
\hline 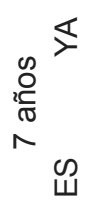 & $\begin{array}{l}\theta \\
\theta\end{array}$ & $r$ & - & $\begin{array}{l}N \forall m \\
N \sim N\end{array}$ & - & $\begin{array}{l}n \sim \\
m \sim c\end{array}$ & - & $\forall$ & $\begin{array}{c}+ \\
\theta\end{array}$ \\
\hline 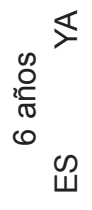 & $\begin{array}{l}A \\
A \\
A\end{array}$ & - & - & $\begin{array}{l}n+m \\
m \forall m\end{array}$ & - & $\begin{array}{l}A m \\
A m c\end{array}$ & & $\begin{array}{c}\theta \\
\theta\end{array}$ & $\begin{array}{c}+ \\
+\end{array}$ \\
\hline 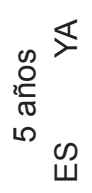 & $\begin{array}{l}\theta \\
\theta\end{array}$ & - & $\sim$ & $\begin{array}{l}m+m \\
N \forall m\end{array}$ & $\forall$ & $\begin{array}{l}A d r \\
\theta\end{array}$ & & $\begin{array}{l}+ \\
\theta\end{array}$ & $\begin{array}{l}\checkmark \\
\nabla\end{array}$ \\
\hline 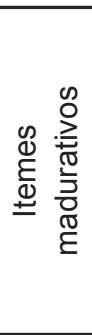 & 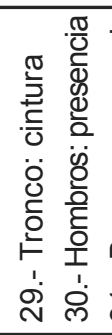 & 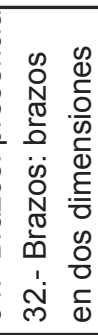 & 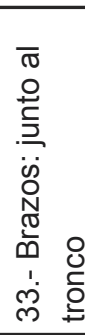 & 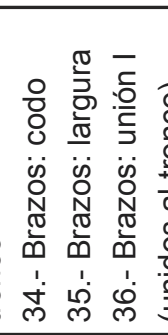 & 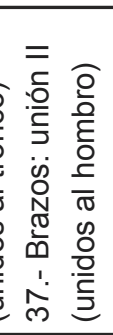 & 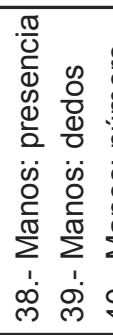 & 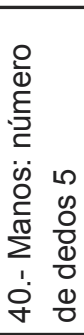 & 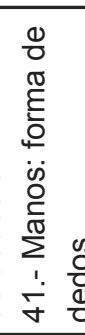 & \\
\hline
\end{tabular}




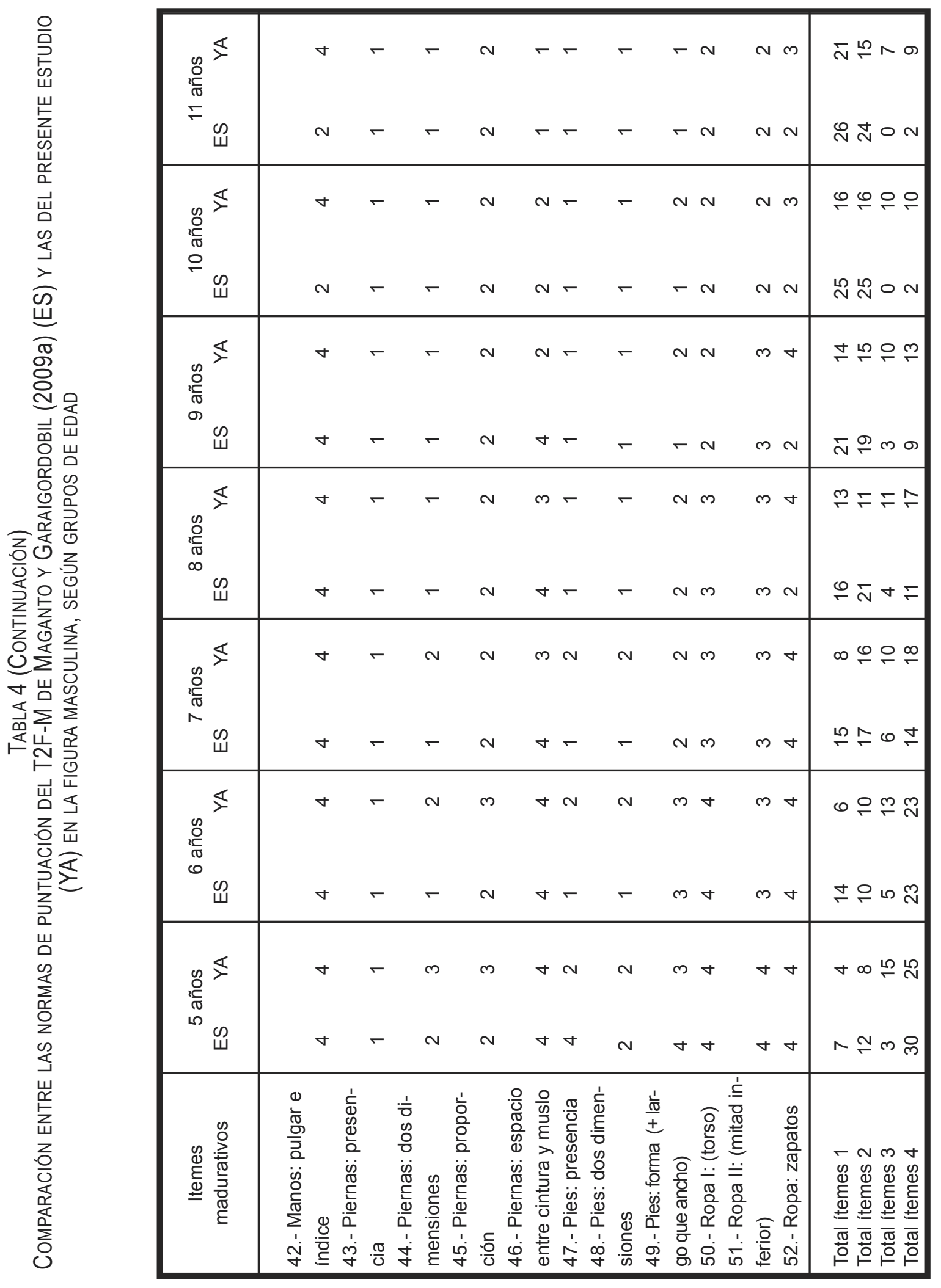


Fernández Nistal, Tuset Bertran y Ochoa Avila

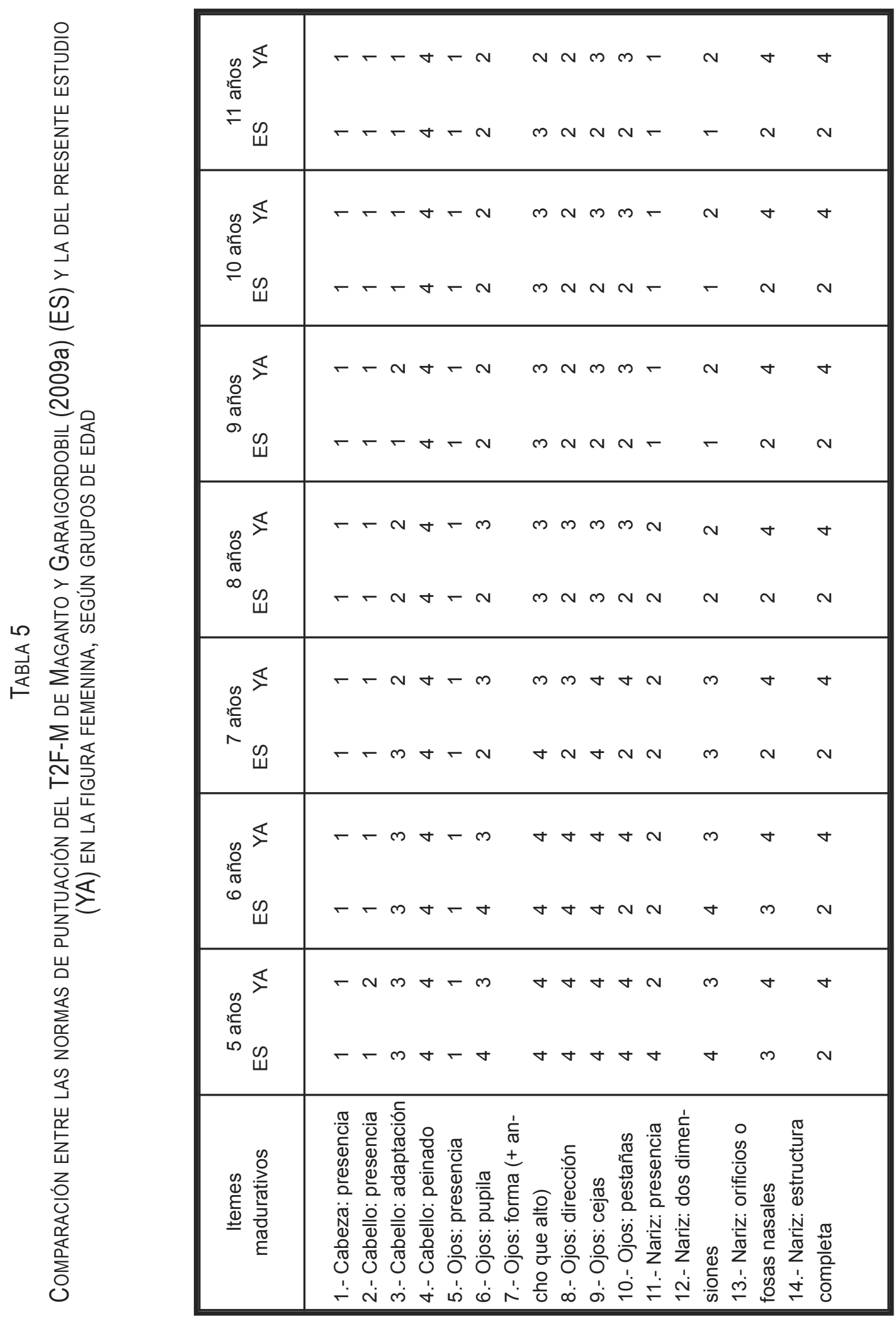




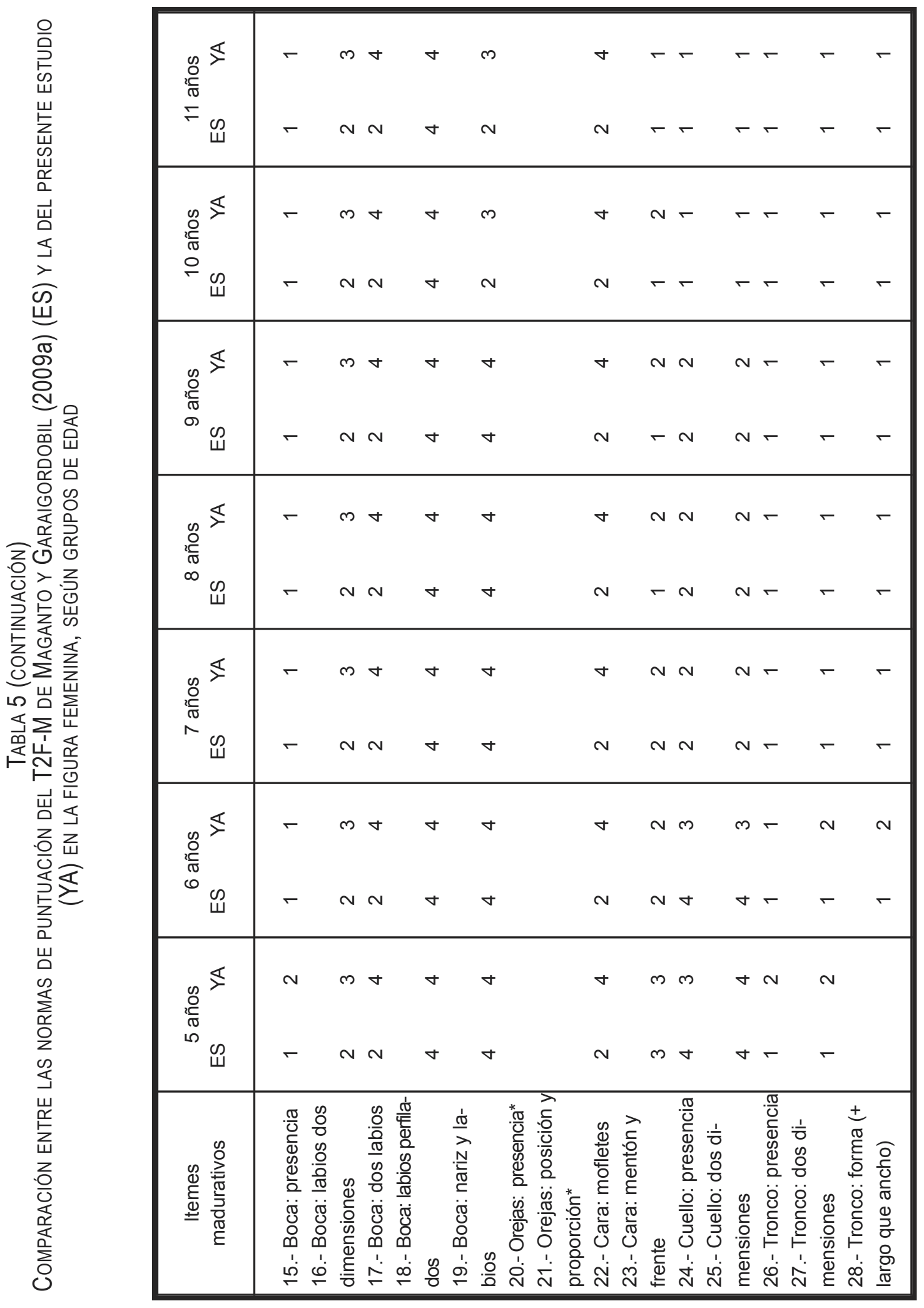




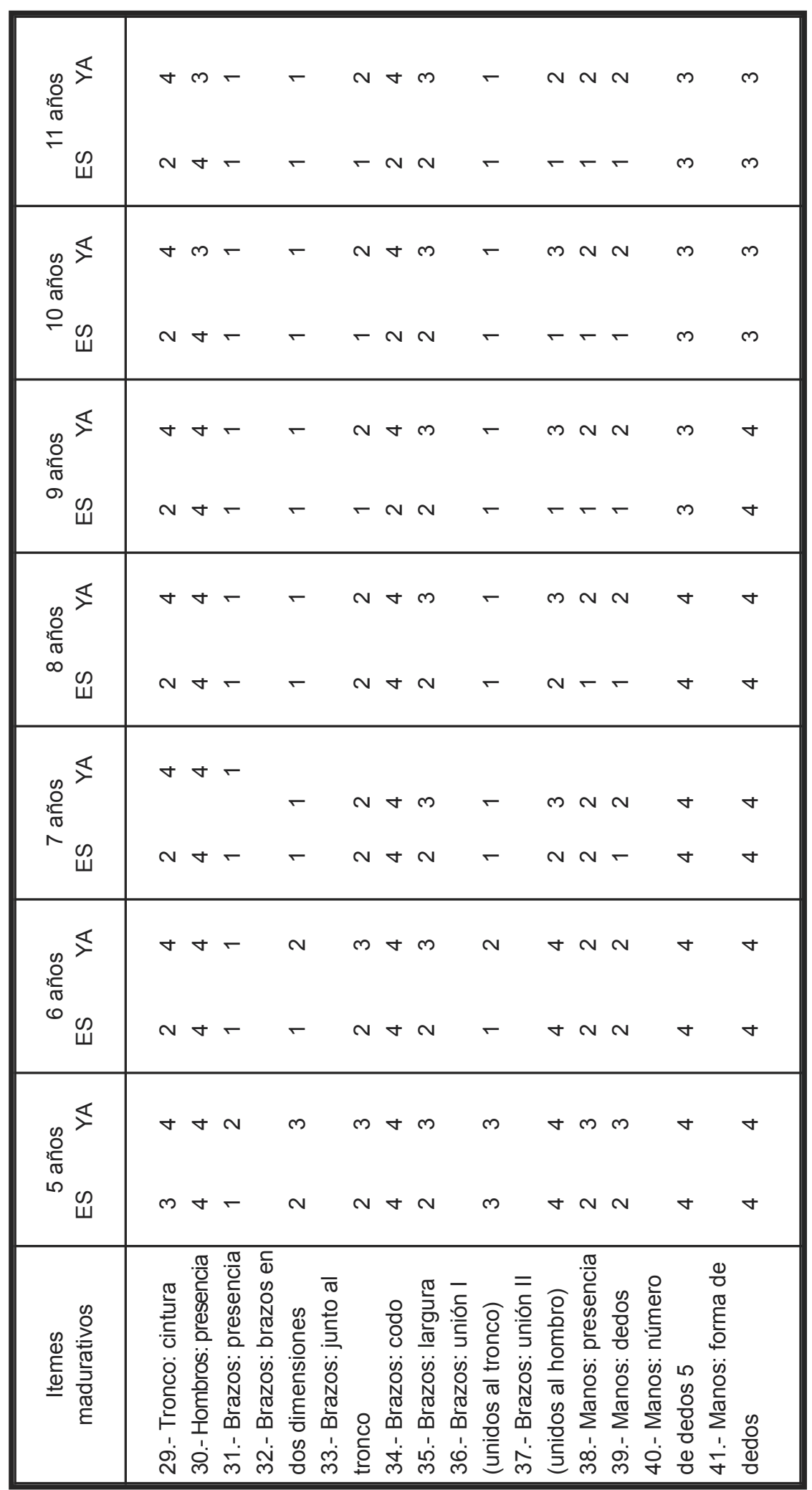

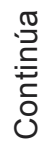




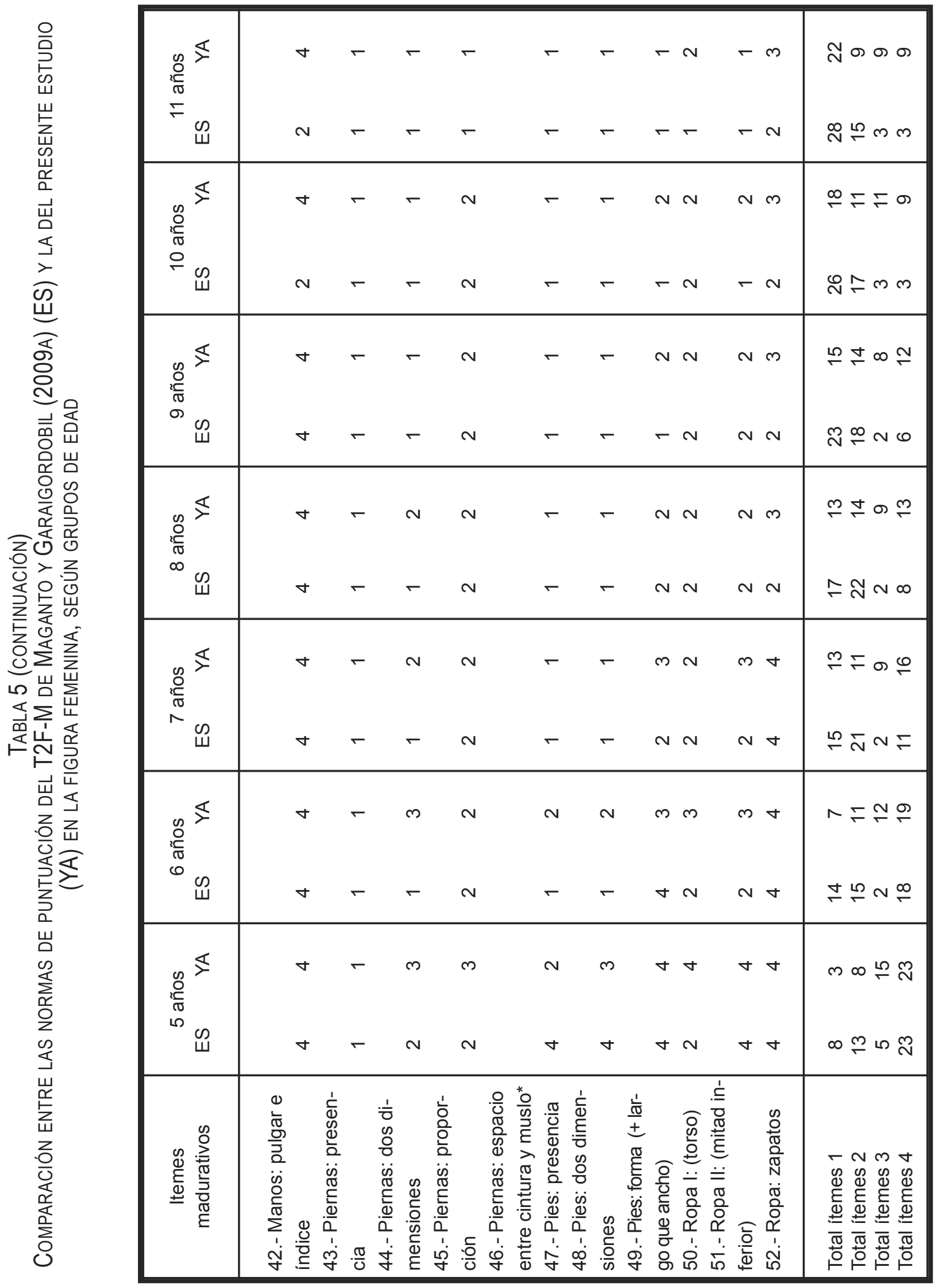




\begin{tabular}{|c|c|c|}
\hline $\begin{array}{l}\stackrel{\infty}{2} \\
\stackrel{2}{\pi} \\
\stackrel{2}{\leftarrow}\end{array}$ & 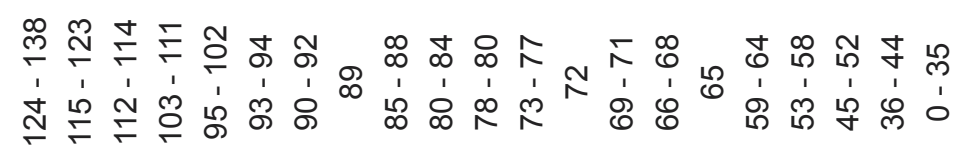 & N \\
\hline $\begin{array}{l}\stackrel{\infty}{2} \\
\stackrel{2}{\pi} \\
\stackrel{2}{0}\end{array}$ & 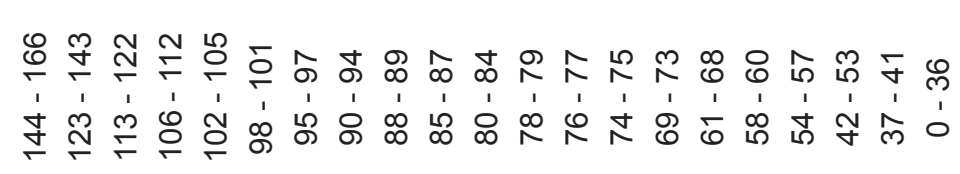 & 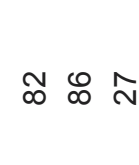 \\
\hline 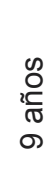 & 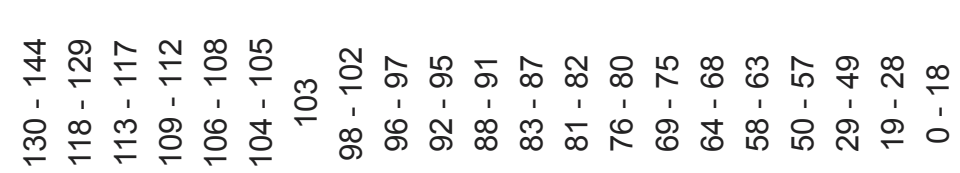 & $\stackrel{\infty}{\infty} \stackrel{\infty}{N}$ \\
\hline 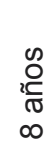 & 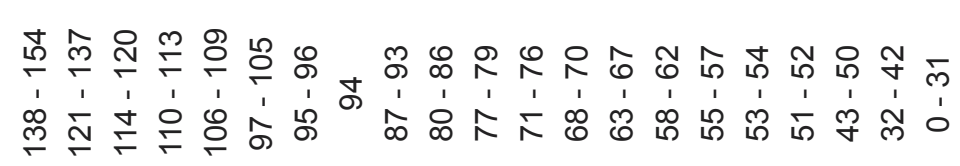 & $\bar{\infty} \stackrel{\infty}{\infty} \stackrel{\infty}{\sim}$ \\
\hline 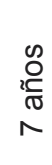 & 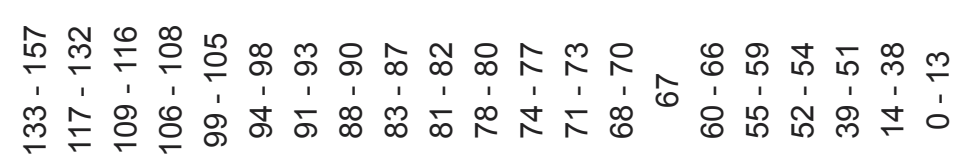 & $\stackrel{\infty}{\circ} \approx$ \\
\hline $\begin{array}{l}\mathscr{0} \\
\substack{\mathbb{T} \\
\pi} \\
0\end{array}$ & 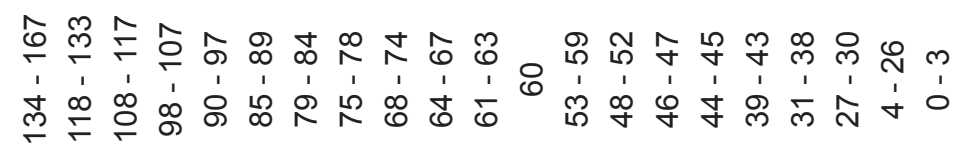 & \& \\
\hline 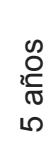 & 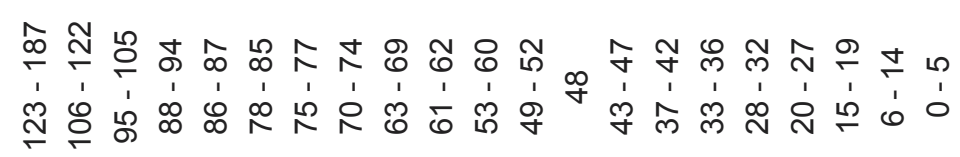 & $\stackrel{\circ}{\circ} \widetilde{\sigma}$ \\
\hline 0 & 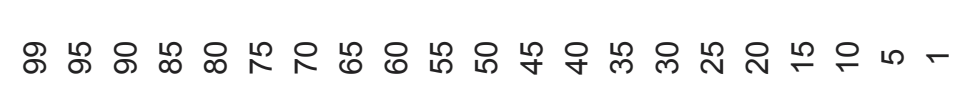 & $z \Sigma$ 占 \\
\hline
\end{tabular}




\section{REFERENCIAS BIBLIOGRÁFICAS}

Abell, S.C., Von Briesen, P.D. \& Watz, L.S. (1996). Intellectual evaluations of children using Human Figure Drawings: An empirical investigation of two methods. Journal of Clinical Psychology, 52(1), 67-74. http://dx. doi.org/10.1002/(SICI)1097-4679(199601) $52: 1<67::$ AID-JCLP9>3.0.CO;2-T

Abell, S.C., Wood, W. \& Liebman, S.J. (2001). Children's human figure drawings as measures of intelligence: The comparative validity of three scoring systems. Journal of Psychoeducational Assessment, 19, 204-215. http://dx. doi.org/10.1177/073428290101900301

Aikman, K.G., Belter, R.W. \& Finch, A.J. (1992). Human figure drawings: Validity in assessing intellectual level and academic achievement. Journal of Clinical Psychology, 48, 114-120.

American Educational Research Association (AE RA), American Psychological Association (APA) \& National Council on Measurement in Education (NCME). (1999). Standards for educational and psychological testing. Washington, DC: American Psychological Association.

Anastasi, A. \& Urbina, S. (1998). Tests psicológicos [Psychological tests]. Estado de México: Prentice Hall.

Archer, R.P., Maruish, M., Imhof, E.A. \& Piotrowski, C. (1991). Psychological test usage with adolescent clients: 1990 survey findings. Professional Psychology: Research and Practice, 22(3), 247-252.http://dx.doi.org/10.103 7/0735-7028.22.3.247

Bandeira, D.R., Costa, A. \& Arteche, A. (2008). Estudo de validade do DFH como medida de desenvolvimento cognitivo infantile [The DFH as a valid measure of children's cognitive development]. Psicología: Reflexao e Crítica, 2(2), 27-56. http://dx.doi.org/10.15 90/S0102-79722008000200020
Carreras, M.A., Uriel, F. \& Fernández, M. (2013). Actualizaciones en el análisis de ítemes madurativos del Dibujo de la Figura Humana en niños escolarizados de Buenos Aires [Maturational items of Human Figure Drawing Test in scholars from Buenos Aires: A revision study]. Interdisciplinaria, 30(1), 101-118.

Cashel, M.L. (2002). Child and adolescent psychological assessment: Current clinical practices and the impact of managed care. Professional Psychology: Research and Practice, 33(5), 446-453. http://dx.doi.org/10.1037//0 735-7028.33.5.446

Casullo, M.M. (1988). El Test Gráfico del Dibujo de la Figura Humana. Normas regionales. [The Graphic Human Figure Drawing Test. Regional norms]. Buenos Aires: Guadalupe.

Consejo Nacional de Población (CONAPO). (2010). Índice de marginación por localidad 2010 [2010 index of marginalization by locality]. México, D.F.: CONAPO. Recuperado el 15 de agosto de 2013 de http://www.conapo. gob.mx.

Costenbader, V., Rohrer, A.M. \& Difonzo, N. (2000). Kindergarten screening: A survey of current practice. Psychology in the Schools, 37, 323-332. http://dx.doi.org/10.1002/15206807(200007)37:4<333::AID-PITS3>3.0.C $\mathrm{O} ; 2-\mathrm{M}$

Cox, M. (1993). Children's drawings of the human figure. Hillsdale: LEA.

Dennis, W. (1966). Goodenough scores, art experience, and modernization. Journal of Social Psychology, 68, 211-228. http://dx.doi.o $\mathrm{rg} / 10.1080 / 00224545.1966 .9919684$

Diario Oficial (2010). Instituto Nacional de Lenguas Indigenas. Programa de revitalización, fortalecimiento y desarrollo de las lenguas indigenas nacionales 2008-2012 [National Institute of Indigenous Languages. Program of revitalization, strengthening and development of the national indigenous languages 2008- 
2012]. PINALI. Recuperado el 21 de agosto de 2013 de http://site.inali.gob.mx/pdf/02_JU L_ PINALI-2008-2012.pdf

Esquivel, F., Heredia, C. \& Lucio, E. (2007). Psicodiagnóstico clínico del niño [Child clinical psychodiagnostic] (3a. ed.). México DF: El Manual Moderno.

Fábila, A. (1978). Las tribus Yaquis de Sonora. Su cultura y anhelada autodeterminación [The Yaqui tribes of Sonora. Their culture and long desired self-determination]. México DF: Instituto Nacional Indigenista.

Flanagan, R. \& Motta, R.W. (2007). Figure drawings: A popular method. Psychology in the Schools, 44(3), 257-270. http://dx.doi.org/10. 1002/pits.20221

Flores-Mendoza, C., Alves, M., Abad, F. \& Lelé, A. (2010). O que mede o desenho da figura humana? Estudos de validade convergente e disciminante [What the human figure drawing test measure? Convergent and discriminant validity study]. Boletín de Psicologia, 60(132), 32-56.

Flynn, J. R. (2007). Beyond the flynn effect. En J.R. Flynn (Ed.), What is intelligence?: Beyond the flynn effect (pp. 4-47). Cambridge, MA: Cambridge University Press.

Gayton, W.F., Tavormina, J., Evans, H.E. \& Schuh, J. (1974). Comparative validity of Harris' and Koppitz' scoring systems for human-figure drawings. Perceptual and Motor Skills, 39, 369370. http://dx.doi.org/10.2466/ pms.1974.39.1. 369

Gonzales, E. (1982). A cross-cultural comparison of the developmental items of five ethnic groups in the southwest. Journal of Personality Assessment, 46, 26-31. http://dx.doi.org/10.12 07/s15327752jpa4601_6

González, A. (1988). Observaciones preventivas en relación a fuentes bibliográficas de habla hispana que refieren el manejo del Dibujo de la Figura Humana en los niños de Elizabeth M. Koppitz [Cautionary remarks regarding Hispanic literature sources referring to handling of the Human Figure Drawing in children by Elizabeth M. Koppitz]. Revista Mexicana de Psicología, 5, 73-81.

Goodenough, F.L. (1926). Measurement of intelligence by drawings. New York: Harcourt, Brace and World.

Harris, D.B. (1991). El Test de Goodenough, revisión, ampliación y actualización [The Goodenough Test. Review, expansion and updating] (2a. ed.). Barcelona: Paidós.

Hutz, C.S. \& Antoniazzi, A.S. (1995). O desenvolvimento do Desenho da Figura Humana em crianças de 5 a 15 anos de idade: Normas para avaliação [The development of the Human Figure Drawing in children 5-15 years of age: Guidelines for evaluation]. Psicologia: Reflexão e Crítica, 8(1), 3-18.

Jegede, R.O. \& Bamgboye, E.A. (1982). A longitudinal study of intelectual maturity in Nigerian children. The Journal of Social Psychology, 117, 299-300. http://dx.doi.org/10.1 080/00224545.1982.9713441

Koppitz, E.M. (1975). The Bender Gestalt Test for young children. Vol. 2: Research and applications, 1963-1973. New York: Grune \& Stratton.

Koppitz, E.M. (2002). El dibujo de la figura humana en los niños [The human figure drawing in children] (12a. ed.). Buenos Aires: Guadalupe. (Trabajo original publicado en 1965).

Laosa, L.M., Swartz, J.D. \& Díaz Guerrero, R. (1974). Perceptual-cognitive and personality development of Mexican and Anglo-American children as measured by human figure drawing. Developmental Psychology, 10(1), 131139. http://dx.doi.org/10.1037/h0035546

Machover, K. (1949). Personality proyection in the drawing of the human figure. Sprinfield Ill.: Charles C. Thomas. 
Maganto, C. \& Garaigordobil, M. (2009a). Test del Dibujo de Dos Figuras Humanas (T2F) [Two Human Figures Test (T2F)]. Madrid: TEA Ediciones.

Maganto, C. \& Garaigordobil, M. (2009b). El diagnóstico infantil desde la expresión gráfica: El Test de Dos Figuras Humanas (T2F) [Child diagnosis by means of graphic expression: The Two Human Figures Test (T2F)]. Clínica y Salud, 20, 237-248.

Martínez, J.A. (Coord.) (2010). Historia regional de Sonora [Sonora regional history]. Hermosillo: Colegio de Bachilleres del Estado de Sonora.

Martlew, M. \& Connolly, K.J. (1996). Human Figure Drawings by Schooled and Unschooled Children in Papua New Guinea. Child Development, 67, 2743-2762.

Mehryar, A.H., Tashakkori, A., Yousefi, F. \& Khajavi, F. (1987). The application of the Goodenough-Harris Draw-A-Man Test to a group of Iranian children in the city of Shiraz. British Journal of Educational Psychology, 57, 401-406. http://dx.doi.org/10.1111/j.2044-827 9.1987.tb00866.x

Merino, C., Honores, L., García, W. \& Salazar, G. (2007). Una evaluación normativa de los ítems evolutivos en el dibujo de la figura humana de Koppitz [A normative assessment of developmental items of the Koppitz Human Figure Drawing]. Nuevos Paradigmas, 1(1), 77-95.

Moctezuma, J.L. (2007). Yaquis. México DF: Comisión Nacional para el Desarrollo de los Pueblos Indígenas.

Motta, R.W., Little, S.G. \& Tobin, M.I. (1993). The use and abuse of human figure drawings. School Psychology Quarterly, 8(3), 162-169.

Naglieri, J.A. (1988). DAP: Draw a person: A quantitative Scoring System Manual. San Antonio: The Psychological Corporation. http:// dx.doi.org/10.1207/s15326985ep2402_4
Ozer, S. (2009). Turkish children's human figure drawings: Can we borrow norms? Educational Psychology, 29(6), 701-712. http://dx.doi.org/ 10.1080/01443410903210387

Pfeffer, K. \& Olowu, A. (1986). Effects of socioeconomic differences on the sophistication of Nigerian children's human figure drawings. Perceptual and Motor Skills, 62, 771-774. http://dx.doi.org/10.2466/pms.1986.62.3.771

Raven, J.C. (1993). Test de Matrices Progresivas. Cuaderno de Matrices, Escala Coloreada. Series $A$, $A b$ y $B$ [Progressive Matrices Test. Notebook Matrices, Colored Scale. Series A, Ab and B]. Buenos Aires: Paidós.

Raven, J.C. (2001). Matrices Progresivas (SPM, $A P M$ y CPM) [Progressive Matrices (SPM, APM and CPM)] (3ra. ed.). Madrid: TEA Ediciones.

Raven, J.C., Court, J.H. \& Raven, J. (1993). Test de Matrices Progresivas. Escalas Coloreada General y Avanzada. Manual [Progressive Matrices Test. Colored, General, and Advanced Scales. Manual]. Buenos Aires: Paidós.

Reynolds, C.R. \& Hickman, J.A. (2004). Draw-APerson Intellectual Ability Test for Children, Adolescents, and Adults (DAP: IQ). Austin, TX: Pro-Ed.

Rosa, H.R. (2008). Validade do Desenho da Figura Humana na avaliação de Goodenough-Harris e nos indicadores maturacionais de Koppitz em crianças da cidade de São Paulo [Human Figure Drawing's validity for Goodenough Harris Test and Koppitz Maturity Indicators in São Paulo children's]. Boletín de Psicologia, 58(128), 001-014.

Rosa, H.R. \& Alves, I.C.B. (2000). R-2: Teste Não Verbal de Inteligência para Crianças [R-2 Non verbal intelligent test for children]. Manual. São Paulo: Vetor Editora.

Rueda, F.J.M. \& Sisto, F.F. (2006). Estudo sobre as categorías de interpretação das Matrizes Colo- 
ridas de Raven e DFH Scala Sisto [Study of the Raven Colored Matrices interpretation categories and DFH-Escala Sisto]. Aletheia, 23, 1726.

Secretaría de Educación y Cultura (2012). Sistema de Información, Control y Registro Escolar de Sonora (SICRES) [Information and Control System and School Registration of Sonora]. Recuperado el 11 de julio de 2012 de http:// www.sicres.gob.mx/

Scott, L.H. (1981). Measuring intelligence with the Goodenough-Harris Drawing Test. Psychological Bulletin, 89(3), 483-505.

Sisto, F.F. (2006). O desenho da Figura HumanaEscala Sisto [Drawing Figure Human - Scale Sisto]. Sao Paulo, S.P: Vetor.

Spicer, E. (1994). Los Yaquis. Historia de una cultura [The Yaquis. A cultural history]. México D F: Universidad Autónoma de México.

Ter Laak, J., de Goede, M., Aleva, A. \& VanRijswijk, P. (2005). The Draw-A-Person Test: An indicator of children's cognitive and socioemotional adaptation? Journal of Genetic Psychology, 166(1), 77-93.
Vedder, P., Van de Vijfeijken, K. \& Kook, H. (2000). Borrowing norms for the human figure drawing test: How to validate this practice? Scandinavian Journal of Educational Research, 44(4), 393-404. http://dx.doi. org/10. 1080/713696680

Vélez, A., Sandoval, C., Ibáñez, M., Talero, C., Fiallo, D. \& Halliday, K. (2011). Validation study of human figure drawing test in a Colombian school children population. The Spanish Journal of Psychology, 14(1), 464477. http://dx.doi.org/10.5209/rev_SJOP.2011. v14.n1.42

Wechsler, D. (1991). Wechsler Intelligence Scale for Children - Third Edition (WISC-III). San Antonio, TX: The Psychological Corporation. Wechsler, S.M. (1998). Adaptação e validação do Desenho da Figura Humana para crianças brasileiras [Adaptation and validation of the Human Figure Drawing for Brazilian children]. Revista Iberoamericana de Diagnóstico y Evaluación Psicológica, 4, 47-64.

Fecha de recepción: 12 de febrero de 2014 Fecha de aceptación: 10 de junio de 2014 